\title{
Real-time in situ observation of shear modulus evolution during Ostwald ripening of colloidal crystallization
}

\author{
Hongwei Zhou ${ }^{\mathrm{a}}$, Shenghua $\mathrm{Xu}^{\mathrm{a}, \mathrm{b}, *}$, Zhiwei Sun ${ }^{\mathrm{a}}$

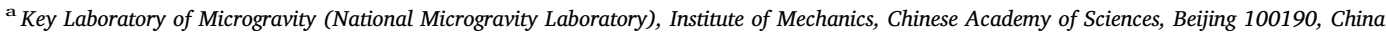 \\ ${ }^{\mathrm{b}}$ School of Engineering Science, University of Chinese Academy of Sciences, Beijing 100149, China
}

\section{A R T I C L E I N F O}

Communicated by R. Fornari

Keywords:

A1. Colloidal crystal

A1. Crystallization

A2. Ostwald ripening

A1. Shear modulus

\begin{abstract}
A B S T R A C T
Real-time in situ observation of evolution of shear modulus during the crystallization and ripening of colloidal particles suspensions was conducted by using reflection spectrum and torsional resonance spectroscopy simultaneously. According to the structural information obtained by reflection spectrum, after the completion of nucleation, the crystallization can be basically divided into four stages: crystallization-dominated stage, crystallization and ripening coexistence stage, ripening-dominated stage and crystallization completion stage. Colloidal particles volume fraction significantly affects the duration of each stage. Our experiments showed that the ripening process is the main cause for the improvement of the mechanical strength (the increase of shear modulus). This observation is supposed to be associated with the diminishing of crystal interface as the result of the increase in crystallites size and the decrease in crystallites number during the ripening process. In addition, a theoretical analysis is given briefly on the softening effect induced by nonaffinity of disordered particles in interface region.
\end{abstract}

\section{Introduction}

Ostwald ripening is a classical physical phenomenon observed in many fields like metals [1-4], protein crystals [5,6], inorganic materials [7-9], and emulsions $[10,11]$. In principle, it is a spontaneous process driven by chemical potential differences among different sized particles. Specifically, larger crystallites grow at the expense of smaller ones which have a higher solubility than the large ones. Ostwald ripening has been adopted as a general synthesis strategy to prepare porous or hollow materials [12-15]. On the other hand, ripening resulting particle/crystallites size distribution changes will inevitably influence the property and performance of the materials. For instance, in industrial catalysis, sintering caused by coarsening or ripening of nanoparticles is one main cause of catalyst deactivation [16-18]. In nanocrystalline metals and alloys, it is well known grain size have a significant effect on the mechanical properties including rigidity, hardness and ductility $[19,20]$. Therefore, it would be of great interest to explore the relationship between the properties of the material and its internal structure like grain size and number, grain boundary, texture et al. With this regard, simultaneously in situ measuring mechanical properties and internal structure of materials is desirable. However, such a measurement is very difficult to implement for atomic or molecular systems because the characteristic size scale and time scale of them are very shortly.

Colloidal suspensions, as highly amplified atoms both in temporal scale and spatial scale, have been served as an ideal model to study many phenomena and processes in condensed matter physics because of the analogy between atomic and colloidal system [21-25]. Particularly, the regulation of the inter-particles interactions, nucleation and phase behavior of colloidal systems can be conveniently controlled by experimental parameters. Much larger temporal and spatial scales of colloidal systems make it possible to adopt a wider range of measurement methods, especially non-invasive optical methods.

For the determination of colloidal crystals structure and structural parameter, Kossel line [26,27], static light scattering (SLS) [28,29] and ultra-small-angle X-ray scattering (USAXS) [30,31] are the mainly used methods. Recently, reflection spectrum (RS) has also been employed $[32,33]$. Due to the high sample rate, RS has great advantage in studying a dynamic process. Moreover, RS is easily coupled with other characterization technique to acquire general information during crystallization and phase transition.

Modulus is a basic mechanical parameter of colloidal crystals. At the microscopic level, modulus is associated with pair potential of interaction between colloidal particles [34,35]. Naturally, interaction

\footnotetext{
* Corresponding author at: Key Laboratory of Microgravity (National Microgravity Laboratory), Institute of Mechanics, Chinese Academy of Sciences, Beijing 100190, China.

E-mail address: xush@imech.ac.cn (S. Xu).
} 
parameters such as particle number density, effective charge number and salt concentration should all have direct impacts on the shear modulus. Additionally, other factors including crystalline morphology, stability and homogeneity also influence shear modulus [35-37]. Recently, shear modulus of defective crystals with vacancies [38] and the continuity of shear modulus when colloidal crystals approaching to the melting [39] were studied within the framework of non-affine lattice dynamics, these results implying that besides the standard Born-Huang affine contribution, softening effect induced by nonaffinity of disordered particles must be taken into account when we study the elasticity of colloidal crystals $[40,41]$. More specifically, interface regions of materials have less contribution to modulus than that in the interior due to the defect of inversion-symmetry (the broken spatial inversionsymmetry) of particles near the interface, resulting a reduction of the overall modulus.

Comparing with traditional atomic materials, shear modulus of colloidal crystals have some distinct characteristics. First, the magnitude of modulus is only a few $\mathrm{Pa}$, which is much smaller than their atomic counterpart (on the order of GPa). Weakly shaking or shearing can melt the crystals. Second, colloidal crystals coexist with the surrounding solvent, which results in the invalid of direct tensile and compressive testing. To resolve this problem, several methods including gravitational sedimentation equilibrium method [42] (method 1), Torsional Resonance Spectroscopy [35] (TRS, method 2) and video microscope method [43] (method 3) have been developed. Method 1 is quasi-static and cannot be used in dynamic process, method 2 and method 3 can use in dynamic process, but method 3 is limited in twodimensional colloidal solids with larger particle size. Therefore, TRS is a proper candidate to investigate the mechanical properties variations in a dynamic crystallization and ripening process.

In this paper, charged colloidal particles were employed and interparticle interaction was tuned by particles volume fraction to control the crystallization and ripening process. Real time in situ structural and mechanical information of the colloidal system were obtained through associated using RS and TRS methods.

\section{Material and methods}

\subsection{Material}

Polystyrene (PS) spheres with ionizable sulfonic groups on their surface were purchased from Huge Biotechnology (Shanghai, China). The received particles were purified and filtrated again by repeated washing with ultrapure water (UPRLC5DRO UPW system, Relatec, China) in the centrifugation process, and then stored with ion-exchange resin (Amberlite IRN-150, EMD Millipore Corporation, Germany) for further deionization. The diameter $(D)$ and polydispersity index (PDI) of the particle are $100 \mathrm{~nm}, 0.04$ obtained from dynamic light scattering (Brookhaven Instruments Corporation, USA). The effective surface charge number $\left(Z_{\text {eff }}\right)$ determined by measuring the increase of conductivity with particle number density $\left(n_{\mathrm{p}}\right)$ is 484 [44].

\subsection{Measurement methods}

In order to simultaneously obtain structural parameters and mechanical strength of the growing crystallites, two techniques were utilized:

\subsubsection{Reflection spectrometer}

The reflection spectrometer is equipped with a tungsten halogen light source (wavelength: 400-1100 nm, Avalight-HAL, Avantes, Netherlands) and an optic spectrometer (Avaspec-2048, Avantes, Netherlands). The incident light and reflected light are coupled in a same "Y" type optic cable. The functionality of reflection spectrometer is to identify crystal lattice structures and to collect light intensity variations in the crystallization process for further extracting the

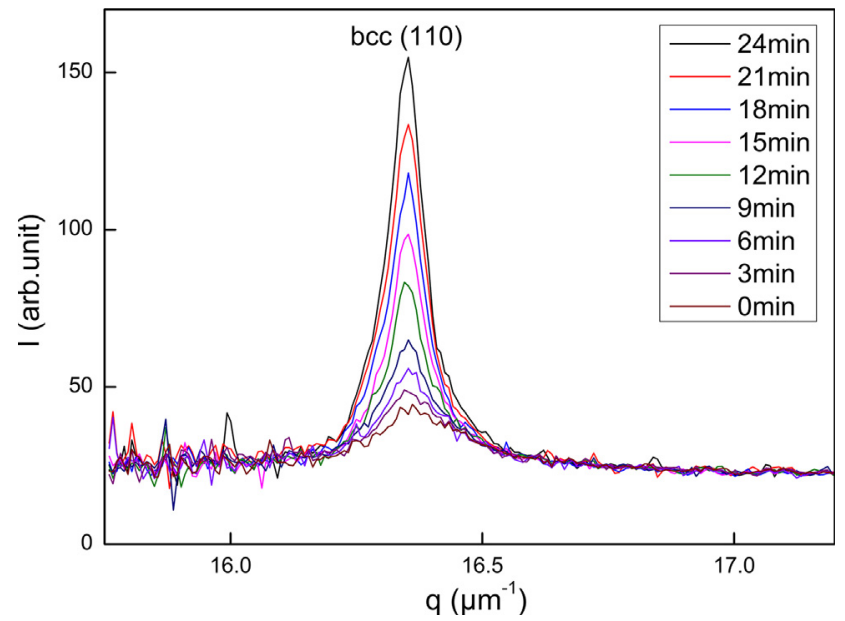

Fig. 1. Time-dependent RS spectra in the crystallization of colloidal suspension with $\Phi=0.5 \%$.

structural parameters. In principle, RS is analogy to the conventional SLS. The only difference is that in SLS method incident wavelength $\lambda$ is fixed and scattering angle $\theta$ is varied, while in RS method $\theta$ is fixed and $\lambda$ is varied. Hence as long as we transfer RS spectra from wavelength space to wave vector space by using $q=(4 \pi \nu / \lambda) \cdot \sin (\theta / 2)$ ( $q$ is wave vector and $\nu$ is refractive index), the same structure analysis method used in SLS can also be applied in RS [32]. Moreover, the time required for RS to complete a measurement is less than that of SLS method by a factor $1 / 1000$, hence it is a more appropriate candidate to conduct real time measurement in the dynamic ripening process.

The following information can be achieved from our RS measurements $[45,46]$ :

(1) Crystal structure. It is determined by the relative position of the reflection peaks [32]. The crystal structure is body centered cubic (bcc) for the two investigated volume fractions $(\Phi=0.5 \%$ and $\Phi=1.5 \%$ ). Typically, the primary peak (bcc (1 110$)$ ) of sample with $\Phi=0.5 \%$ in the crystallization were shown in Fig. 1 . The peak intensity increases with the proceeding of crystallization.

(2) Crystallinity $X(t) . X(t)$ is calculated from the area of the primary peak by $X(t)=c A(t)$, where $c$ is a normalization factor and $A(t)$ is the area of the primary peak when the crystals reach equilibrium state. $X(t)$ represents the fraction of the sample which has become crystalline from liquid, it can be used as an indicator to show the degree of crystallization. $X(t)=0$ means the sample is in liquid state and there is no crystalline state, and $X(t)=1$ indicates all the liquid part has been transformed into crystalline state.

(3) Average crystallites size $L(t)$. $L(t)$ represents the average crystallites size and it is given by $L(t)=2 \pi K / \Delta q(t)$, where $K=1.15$ is the Scherrer constant for crystallites of spherical shape [45], $\Delta q(t)$ is the full width at half maximum (FWHM) of the primary peak.

(4) Crystallites number $\boldsymbol{N}_{c}(t) . N_{c}(t)$ is estimated by $N_{c}(t)=X(t) / L^{3}(t)$. $N_{c}(t)$ reflects the change of crystallites number. Generally, $N_{c}(t)$ keeps constant in crystal growth, and the decrease of $N_{c}(t)$ usually means the starting of ripening because larger crystallites grow at the expense of smaller ones. However, no matter the crystallites are larger or smaller, they are both in crystalline state, so ripening only changes $N_{c}(t)$ and it is irrelevant with $X(t)$.

\subsubsection{Torsional resonance spectroscopy}

This setup is a non-contact technique to determine the shear modulus of colloidal crystals by measuring the resonance frequency. Colloidal crystals in the cylindrical cell undergo forced oscillation which is exerted by the cylindrical cell connected with loudspeaker. The excited periodical oscillation of inter-planar distance of colloidal 


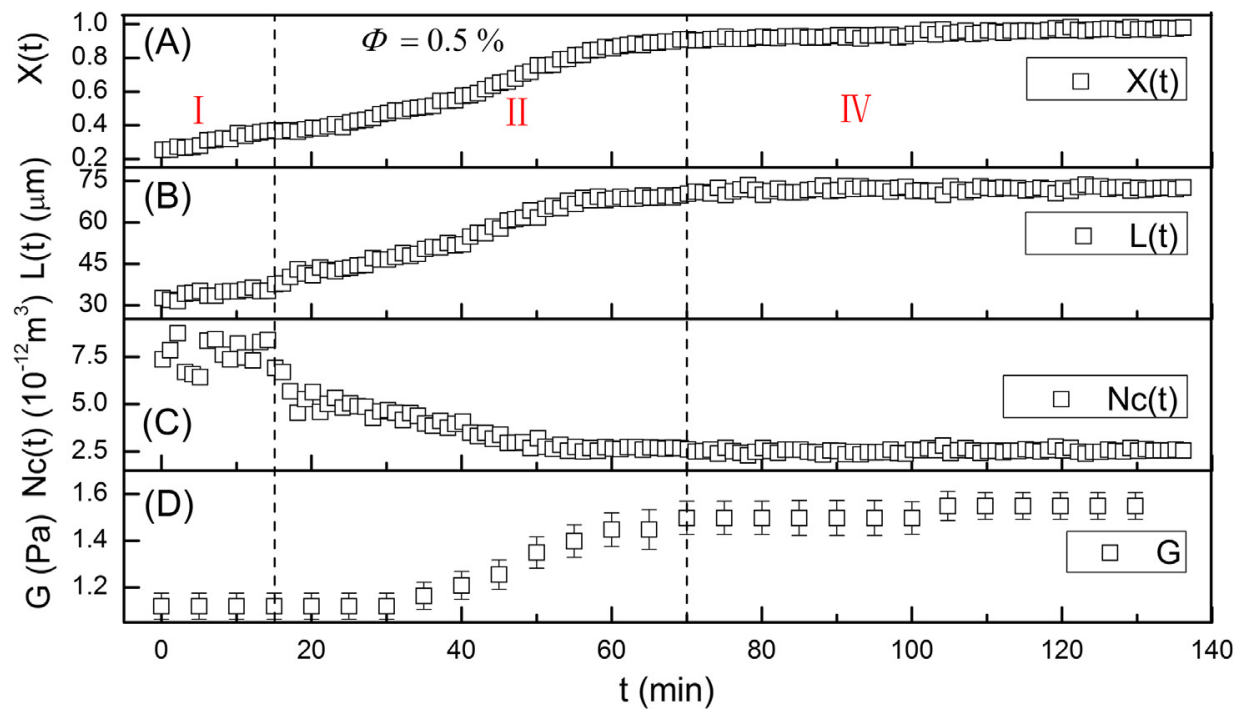

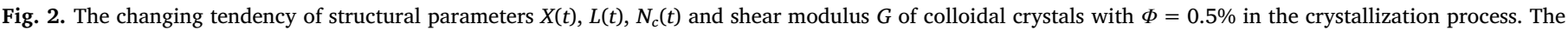
whole process is divided into three stages $I, I I, I V$.

crystals can be reflected by the displacement of Bragg peaks, which is detected by position sensitive detector. When the displacement magnitude reaches its maximum, inherent frequency of the colloidal crystals can be identified by lock-in technique. The shear modulus $G$ can then be calculated from the resonance frequency $\omega$ by the following expression [35]:

$G=\frac{\omega_{j n}^{2} \rho R^{2}}{\mu_{j}^{2}+(n+1 / 2)^{2} \pi^{2} R^{2}}$

where $R$ is the inner radius of the cylindrical vial, $\alpha=R / H$ is the aspect ratio between $R$ and the filling height $H, \rho$ is the mass density of the suspensions, and $\mu_{j}(=3.83,7.01,10.17, \ldots)$ are zeros of the first order Bessel function with $j=1,2,3, \ldots$ and $n=0,1,2, \ldots$ are the number of nodes of the vertical standing wave within $H$.

\subsection{Experimental procedure}

Colloidal suspensions with different volume fractions $\Phi\left(=n_{\mathrm{p}} \cdot \pi D^{3}\right.$ / 6) were prepared by mixing certain amount of latex and pure water in the vials. After adding in some ion-exchange resin the vials were sealed with laboratory film (Parafilm $\mathrm{M}^{\circledR}$, USA) and put on the rotation stage to deionization for several days. At the beginning of the measurement, the deionized sample was pumped into a cylindrical quartz tube with a syringe to filtrate the resin, and the tube was plugged with a silicone stopper quickly and sealed with laboratory film. Both of the two employed volume fractions $(0.5 \%, 1.5 \%)$ are larger than the critical crystallization volume fraction (about $0.2 \%$ ), making both cases in crystalline state. After shaking or shearing to melt the crystals, the tube was inserted into the observation hole quickly, and RS and TRS were turned on immediately to collect the structural and mechanical information in the recrystallization process. It should be pointed out that our measurements are restricted because both RS and TRS methods rely on light interference pattern resulted by Bragg diffraction from crystal lattices. That is, such measurements are valid only after a certain size of the crystal grain has been formed, or measurements can be carried out only after the completion of nucleation. In view of this situation, the beginning time of our measurements (we set it as $t=0$ ) had to be delayed for a short period of time (approximate $1 \mathrm{~min}$ ) than the actual starting time of crystallization.

\section{Results and discussion}

The whole crystallization process can be roughly divided into two stages: crystallization subprocess (including nucleation and crystal growth) and ripening subprocess. However, these two subprocesses are not always clearly separated, that is, the two may overlap each other, rather than one following the other. As characterized by the parameters $X(t), N_{c}(t), L(t)$, after the completion of nucleation, the crystallization process in our experiment can be roughly divided into four stages:

I. Crystallization-dominated stage. At this stage, $X(t)$ and $L(t)$ continue to increase while $N_{c}(t)$ remains basically unchanged. These parameters indicate that there is basically no more new crystallites to come up, nor more old ones to disappear, only crystallites themselves grow up.

II. Crystallization and ripening coexistence stage. At this stage, both crystallization and ripening are going concurrently, characterized by that $X(t)$ and $L(t)$ continue to increase, while $N_{c}(t)$ is decreasing.

III. Ripening-dominated stage. This stage is characterized by that $L(t)$ yet increases but $X(t)$ has already reached its maximum and basically keeps unchanged. Meanwhile, $N_{c}(t)$ still decreases, implying that larger crystallites increase in size at the expense of smaller ones. Note, at this stage, the size of the crystallites is also increasing, but the mechanism behind is different from stage $I$, in which the source of crystallites growth is disordered particles.

IV. Crystallization completion stage. At this stage, $X(t), N_{c}(t)$ and $L(t)$ have no further variations.

Our experiments showed that the duration of each stage is significantly affected by colloidal particles volume fraction. For lower volume fraction ( $\Phi=0.5 \%$ ), stages $I, I I, I V$ were observed, while for higher volume fraction ( $\Phi=1.5 \%$ ), stages II, III, IV were observed only.

For $\Phi=0.5 \%$, the variations of structural parameters $X(t), L(t)$, $N_{c}(t)$ and also shear modulus $G$ are shown in Fig. 2(A)-(D), respectively. From these trends we can see that the whole crystallization process seems to have roughly three stages: I, II, IV. Synchronous mechanical properties measurements show shear modulus $G$ is only related to ripening (characterized by the deduction of $N_{c}(t)$ ), not to crystallization (reflected by the increase of $X(t)$ ). For instance, though $X(t)$ increases from 0.2 to 0.4 in stage $I, G$ remains unchanged. But once $N_{c}(t)$ begins to decrease, the following increases of $G$ will be observed. In stage $I I, N_{c}(t)$ continuously decreases and $G$ increases steadily along with the proceeding of ripening. At last, all structural parameters and modulus have 


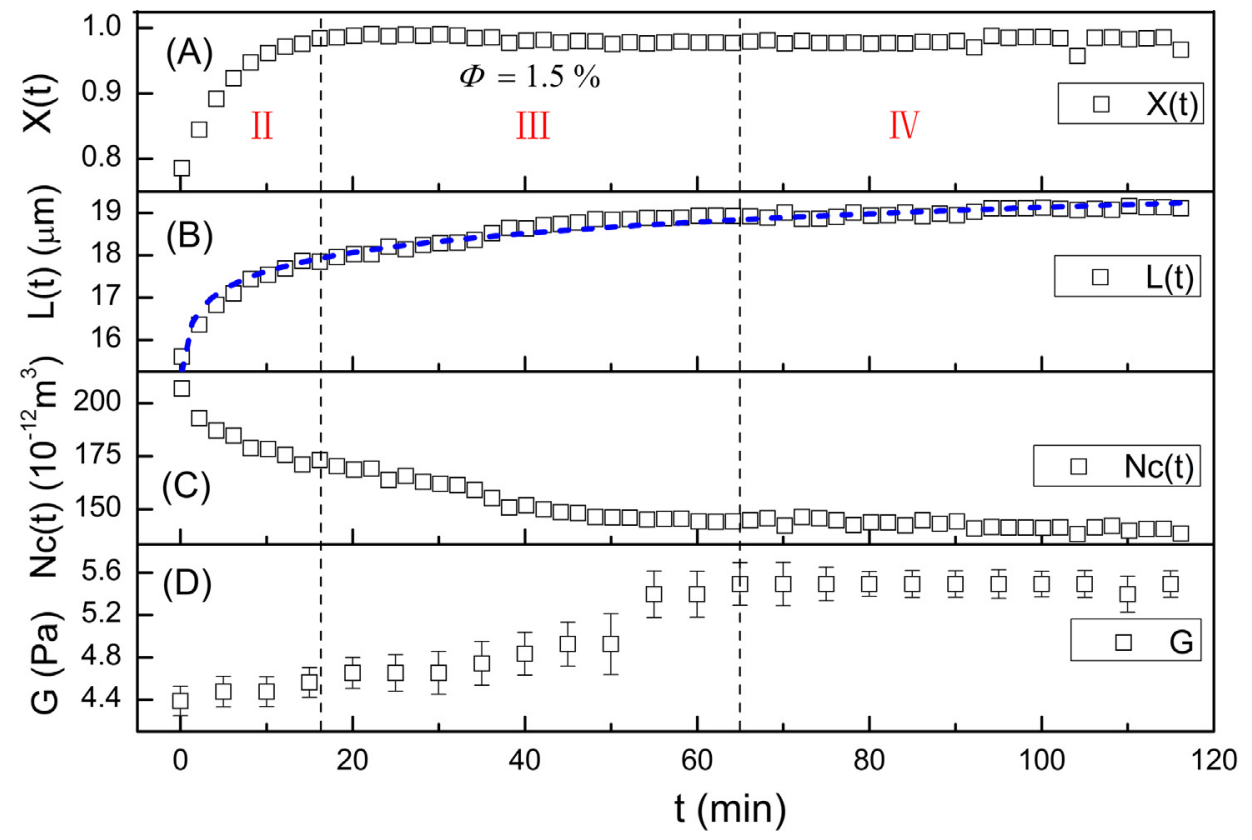

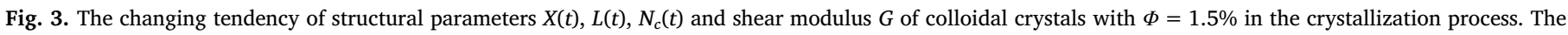
whole process is divided into three stages II, III, IV.

no further variations in the stage of crystallization completion.

Similarly, for $\Phi=1.5 \%$ shown in Fig. 3(A)-(C), after the completion of nucleation, the crystallization process are also divided into three stages II, III, IV. However, stage I disappears while stage III appears compared with $\Phi=0.5 \%$. Because with increasing $\Phi$, nucleation and growth rate increase greatly, stage $I$ can finish very quickly. We can see $X(t) \approx 0.8$ at $t=0$ (note $t$ is the starting time of measurement), which indicates that most of the disordered-particles (liquid) have already formed ordered-grains (crystal) before our actual measurement started. High crystallinity is also a prerequisite for the ripening process. Therefore, the first process that can be observed is already in stage II for $\Phi=1.5 \%$. Additionally, stage $I I I$ appears in the case of $\Phi=1.5 \%$, while it is not found in the case of $\Phi=0.5 \%$. We note such a "ripeningdominated" process has also been found in poly-disperse hard-spherelike colloids [47]. The $L(t)-t$ relation can be well fitted by power law with the exponent 0.036 , which is significantly smaller than the value of 0.33 expected for Ostwald ripening [48]. However, this value is close to the value reported in Ref. [43]. An extended interface region surrounding the growing crystallites is believed to be the main cause. In the extended interface region, the particle density and structure are in the intermediate between ordered crystal and disordered liquid, and the exchange of particles between ripening crystallites are mediated by the particles fractionation inside the partially crystalline and partially amorphous grain boundaries, hence the ripening kinetics can no longer be expressed only in terms of single particle dynamics, but rather that the efficiency of size fractionation and migration in the particle rearrangement. Modulus measurements (Fig. 3(D)). again show that the increase of $G$ is accompanied by ripening and has nothing to do with the variation of $X(t)$. We can see $G$ starts to increase at the beginning of measurement, no matter $X(t)$ increases (stage $I$ ) or not (stage $I I$ ). Additionally, $G$ seems to increase faster in stage III than stage II.

Microscopically, there are lots of interfaces between crystallites because the formed crystals are usually polycrystalline, and particles depending on their locations fall into two categories: interior particles and interface particles. The interior particles located at ordered lattices are in the equilibrium state of force and have stronger resistance to deformation than interface particles As indicated by Zaccone et al. [38-41], for interface particles nonaffine motions become important because of deteriorated ordering and loss of inversion-symmetry, resulting in a force imbalance on each particle in the affine position of deformed configuration and "local" modulus at the interface is much smaller than in the interior. Such a softening effect caused by interfaces has also been observed in ripening process of colloidal crystals in this study. In our experiments, this effect of softening $\left(G_{s}\right)$ is estimated as $G_{\mathrm{s}} \propto N^{-2 / 3}$, where $N$ is the number of colloidal particles in the crystallite. Considering $N \propto V_{\text {crystallite }} \propto L(t)^{3}$, the relation of $G_{s}$ and $L(t)$ can be simply evaluated as $G_{s} \propto L(t)^{-2}$. As a result, the nonaffine shear modulus $\left(G_{0}, G_{0}=G\right)$ can be approximately expressed as $G_{0}=G_{\mathrm{a}}-G_{\mathrm{s}}=G_{\mathrm{a}}-K \cdot L(t)^{-2}$, where $G_{\mathrm{a}}$ is the affine shear modulus, $K$ is scale coefficient. That is to say, $G_{0}$ is linear with $L(t)^{-2}$, which is verified as shown in Fig. 4. Interestingly, in Fig. 4(B). two different slopes, corresponding to stage II and stage III, exist. For stage II (crystallization and ripening coexistence), the absolute value of slope is smaller than that of stage III (ripening-dominated), implying softening effect is weaker comparing with stage III. The possible reason is that in stage II where ripening parallels with crystallization, interface particles at the surface of the crystallizing crystallites are readily registering into the lattice and they can be seen as quasi-affine particles, so their nonaffine contributions to the modulus is weak. In stage III, ripening dominates the behavior of crystallites, dissolving of the smaller crystallites may enforce the motion of boundary particles, and thus the softening effect becomes remarkable.

Based on the above experimental results, our findings can be outlined below:

(1) The nucleation process proceeds very quickly. As mentioned earlier, there is a short delay (about $1 \mathrm{~min}$ or so) between the time we actually start experimental measurement and the starting time of the crystallization process. Our experimental procedure is unable to check what actually happened in the first minute of the crystallization process. What we can judge is that the crystallinity has reached 0.8 and the number of grains will stop further increase after that. That is, within a minute over $80 \%$ of disordered particles have already become ordered ones composed of crystallites.

(2) After a short period of nucleation process that we can't observe, the crystallization process can be roughly divided into four stages: crystallization-dominated stage, crystallization and ripening coexistence stage, ripening-dominated stage and crystallization 

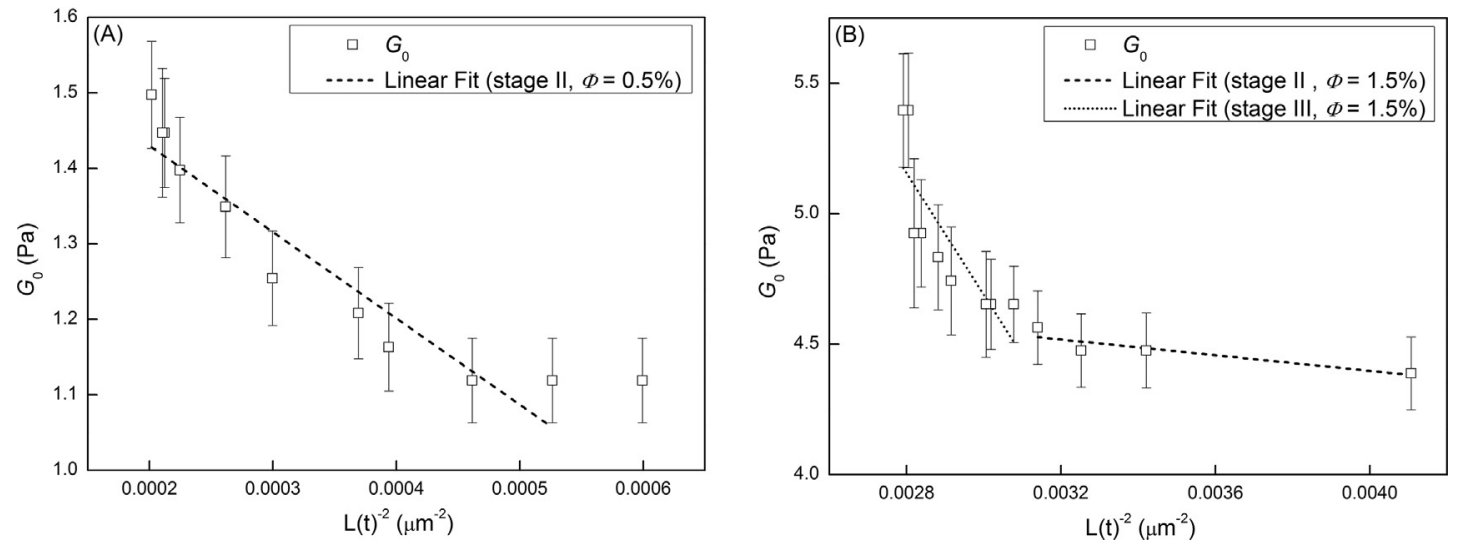

Fig. 4. The relationship of $G_{0}$ and $L(t)^{-2}$ in different stages where ripening exist. (A) Stage $I I, \Phi=0.5 \%$; (B) Stages $I I$ and $I I I, \Phi=1.5 \%$.

completion stage. The duration of each stage is significantly affected by colloidal particles volume fraction. Sample with larger volume fraction has stronger inter-particle interaction because of larger particle density and smaller inter-particle distance. From the point of view of system energy reducing, the driving force to reduce energy of grain boundary for higher volume fraction is larger than that for lower ones, which may result in the difference of stages for samples with different volume fractions. At lower supersaturation (or volume fraction), crystallization-dominated stage can be observed clearly but a separated ripening-dominated stage does not exist. At higher supersaturation the crystallization-dominated stage can't be seen clearly but the ripening-dominated stage does exist alone. Obviously, at lower supersaturation, the low crystallization rate makes it not easy to produce so many crystallites that are apparently different in size, in favor of the ripening process. On the other hand, at higher supersaturation, even from the beginning of crystallization, conditions would have already been conducive to the ripening process. We can infer that with the increase of supersaturation and thus stronger inter-particle interaction, the time of crystallization alone is shortened, and the time of ripening is extending.

(3) The experimental results show that the single crystallization process has little effect on the mechanical strength (represented by the shear modulus $G$ here) of crystallites, and the ripening process is the main cause for the improvement of the mechanical strength. We can understand like this, the structures formed by many small crystallites have very weak resistance to shear due to easy sliding between crystallites. The ripening process makes crystallites become fewer in number but larger in size and thus the decrease of crystal boundaries and interface particle numbers. Therefore, more particles are located in the interior of the crystallites instead of crystal boundaries, and the bonding forces between particles in larger crystallites will improve the shear resistance of structures.

\section{Conclusions}

To investigate the variations of mechanical properties in the solidification of colloidal particle suspensions, RS and TRS were used to conduct real-time in situ measurements. According to the changing tendency of structural parameters $X(t), L(t)$ and $N_{c}(t)$, we suggest that after nucleation the crystallization process may be divided into four stages: crystallization-dominated stage, crystallization and ripening coexistence stage, ripening-dominated stage and crystallization completion stage. The duration of each stage is controlled by colloidal particles volume fraction or supersaturation. Our observations show the trend that with the increase of supersaturation, the time of crystallization alone is shortened and the time of ripening is extending. The experimental results also show that the single crystallization process has little effect on the shear modulus, and the ripening process is the main cause of the increase of shear modulus. Crystal boundary diminishing due to the decreasing of $N_{c}(t)$ and increasing of $L(t)$ may account for this phenomenon. This view is further supported by a theoretical analysis of the softening effect induced by nonaffinity of disordered particles in interface region.

\section{Conflict of interest}

The authors declare no competing financial interests.

\section{Acknowledgements}

This work is supported by the National Natural Science Foundation of China (Grant Nos. 11672295, 11572322 and 11302226).

\section{References}

[1] G. Pia, F. Delogu, Coarsening of nanoporous Au: relationship between structure and mechanical properties, Acta Mater. 99 (2015) 29-38.

[2] M.L. Lescoat, J. Ribis, Y. Chen, E.A. Marquis, E. Bordas, P. Trocellier, Y. Serruys, A. Gentils, O. Kaitasov, Y. de Carlan, A. Legris, Radiation-induced Ostwald ripening in oxide dispersion strengthened ferritic steels irradiated at high ion dose, Acta Mater. 78 (2014) 328-340.

[3] M.C. Flemings, Coarsening in solidification processing, Mater. Trans. 46 (2005) 895-900.

[4] M.C. Zhao, T. Hanamura, H. Qiu, K. Nagai, K. Yang, Grain growth and Hall-Petch relation in dual-sized ferrite/cementite steel with nano-sized cementite particles in a heterogeneous and dense distribution, Scr. Mater. 54 (2006) 1193-1197.

[5] A.M. Streets, S.R. Quake, Ostwald ripening of clusters during protein crystallization, Phys. Rev. Lett. 104 (2010) 178102.

[6] J.K. Baird, R.L. McFeeters, Effects of hydrodynamic convection and interionic electrostatic forces on protein crystallization, Cryst. Growth Des. 13 (2013) 1889-1898.

[7] M. Lin, Z.Y. Fu, H.R. Tan, J.P.Y. Tan, S.C. Ng, E. Teo, Hydrothermal synthesis of $\mathrm{CeO}_{2}$ nanocrystals: ostwald ripening or oriented attachment? Cryst. Growth Des. 12 (2012) 3296-3303.

[8] M. Oliva-Ramirez, M. Macias-Montero, A. Borras, A.R. Gonzalez-Elipe, Ripening and recrystallization of $\mathrm{NaCl}$ nanocrystals in humid conditions, Rsc Adv. 6 (2016) 3778-3782.

[9] M. Tiemann, F. Marlow, J. Hartikainen, O. Weiss, M. Linden, Ripening effects in ZnS nanoparticle growth, J. Phys. Chem. C 112 (2008) 1463-1467.

[10] B. Zeeb, M. Gibis, L. Fischer, J. Weiss, Influence of interfacial properties on Ostwald ripening in crosslinked multilayered oil-in-water emulsions, J. Colloid Interf. Sci. 387 (2012) 65-73.

[11] J. Schmitt, S. Hajiw, A. Lecchi, J. Degrouard, A. Salonen, M. Imperor-Clerc, B. Pansu, Formation of superlattices of gold nanoparticles using Ostwald ripening in emulsions: transition from fcc to bcc structure, J. Phys. Chem. B 120 (2016) 5759-5766.

[12] J. Li, H.C. Zeng, Hollowing Sn-doped $\mathrm{TiO}_{2}$ nanospheres via Ostwald ripening, J. Am. Chem. Soc. 129 (2007) 15839-15847.

[13] R. Shilpa, P. Sharma, A Li-ion storage in morphology tailored porous hollow $\mathrm{Cu}_{2} \mathrm{O}$ nanospheres fabricated by Ostwald ripening, RSC Adv. 6 (2016) 105231-105238.

[14] J.J. Teo, Y. Chang, H.C. Zeng, Fabrications of hollow nanocubes of $\mathrm{Cu}_{2} \mathrm{O}$ and $\mathrm{Cu}$ via reductive self-assembly of CuO nanocrystals, Langmuir 22 (2006) 7369-7377.

[15] G.H. Tian, Y.J. Chen, W. Zhou, K. Pan, Y.Z. Dong, C.G. Tian, H.G. Fu, Facile solvothermal synthesis of hierarchical flower-like $\mathrm{Bi}_{2} \mathrm{MoO}_{6}$ hollow spheres as high 
performance visible-light driven photocatalysts, J. Mater. Chem. 21 (2011) 887-892.

[16] D. Kistamurthy, A.M. Saib, D.J. Moodley, J.W. Niemantsverdriet, C.J. Weststrate Ostwald ripening on a planar $\mathrm{Co} / \mathrm{SiO}_{2}$ catalyst exposed to model fischer-tropsch synthesis conditions, J. Catal. 328 (2015) 123-129.

[17] T.W. Hansen, A.T. DeLaRiva, S.R. Challa, A.K. Datye, Sintering of catalytic nanoparticles: particle migration or Ostwald ripening? ACC Chem. Res. 46 (2013) 1720-1730.

[18] S.R. Challa, A.T. Delariva, T.W. Hansen, S. Helveg, J. Sehested, P.L. Hansen, F. Garzon, A.K. Datye, Relating rates of catalyst sintering to the disappearance of individual nanoparticles during Ostwald ripening, J. Am. Chem. Soc. 133 (2011) 20672-20675.

[19] M.A. Meyers, A. Mishra, D.J. Benson, Mechanical properties of nanocrystalline materials, Prog. Mater Sci. 51 (2006) 427-556.

[20] K.S. Kumar, H. Van Swygenhoven, S. Suresh, Mechanical behavior of nanocrystalline metals and alloys, Acta Mater. 51 (2003) 5743-5774.

[21] A. Yethiraj, A. van Blaaderen, A colloidal model system with an interaction tunable from hard sphere to soft and dipolar, Nature 421 (2003) 513-517.

[22] P. Wette, H.J. Schope, Nucleation kinetics in deionized charged colloidal model systems: a quantitative study by means of classical nucleation theory, Phys. Rev. E 75 (2007) 051405.

[23] T. Palberg, Crystallization kinetics of colloidal model suspensions: recent achievements and new perspectives, J. Phys.: Condens. Matter 26 (2014) 333101.

[24] U. Gasser, Crystallization in three- and two-dimensional colloidal suspensions, J. Phys.: Condens. Matter 21 (2009) 203101.

[25] V.J. Anderson, H.N.W. Lekkerkerker, Insights into phase transition kinetics from colloid science, Nature 416 (2002) 811-815.

[26] T. Shinohara, T. Yoshiyama, I.S. Sogami, T. Konishi, N. Ise, Measurements of elastic constants of colloidal silica crystals by kossel line analysis, Langmuir 17 (2001) 8010-8015.

[27] T. Shinohara, T. Kurokawa, T. Yoshiyama, T. Itoh, I.S. Sogami, N. Ise, Structure of colloidal crystals in sedimenting mixed dispersions of latex and silica particles, Phys. Rev. E 70 (2004) 062401.

[28] P.S. Mohanty, W. Richtering, Structural ordering and phase behavior of charged microgels, J. Phys. Chem. B 112 (2008) 14692-14697.

[29] T. Okubo, K. Kiriyama, N. Nemoto, H. Hashimoto, Static and dynamic light-scattering of colloidal gases, liquids and crystals, Colloid Polym. Sci. 274 (1996) 93-104.

[30] W.L. Vos, M. Megens, C.M. vanKats, P. Bosecke, X-ray diffraction of photonic colloidal single crystals, Langmuir 13 (1997) 6004-6008.

[31] T. Harada, H. Matsuoka, Ultra-small-angle x-ray and neutron scattering study of colloidal dispersions, Curr. Opin. Colloid Interface Sci. 8 (2004) 501-506.

[32] H.W. Zhou, S.H. Xu, Z.W. Sun, X. Du, J.C. Xie, Rapid determination of colloidal crystal's structure by reflection spectrum, Colloids Surf. A 375 (2011) 50-54.

[33] R. Goldberg, H.J. Schope, Opaline hydrogels: Polycrystalline body-centered-cubic bulk material with an in situ variable lattice constant, Chem. Mater. 19 (2007) 6095-6100.

[34] J.F. Joanny, Acoustic shear-waves in colloidal crystals, J. Colloid Interface Sci. 71 (1979) 622-624.

[35] T. Palberg, J. Kottal, T. Loga, H. Hecht, E. Simnacher, F. Falcoz, P. Leiderer Determination of the shear modulus of colloidal solids with high-accuracy, J. Phys. III (4) (1994) 457-471.

[36] H.W. Zhou, S.H. Xu, Z.W. Sun, R.Z. Zhu, Shear moduli in bcc-fcc structure transition of colloidal crystals, J. Chem. Phys. 143 (2015) 144903.

[37] L. Wang, S. Xu, H. Zhou, Z. Sun, F. Xu, Effect of void structures in crystalline structure on the shear moduli of charged colloidal crystals, Colloids Surf. A 516 (2017) 115-120.

[38] J. Krausser, R. Milkus, A. Zaccone, Non-affine lattice dynamics of defective fcc crystals, Soft Matter 13 (2017) 6079-6089.

[39] J. Sprakel, A. Zaccone, F. Spaepen, P. Schall, D.A. Weitz, Direct observation of entropic stabilization of bcc crystals near melting, Phys. Rev. Lett. 118 (2017) 088003.

[40] A. Zaccone, J.R. Blundell, E.M. Terentjev, Network disorder and nonaffine deformations in marginal solids, Phys. Rev. B 84 (2011) 174119.

[41] A. Zaccone, E. Scossa-Romano, Approximate analytical description of the nonaffine response of amorphous solids, Phys. Rev. B 83 (2011) 184205.

[42] R.S. Crandall, R. Williams, Gravitational compression of crystallized suspensions of polystyrene spheres, Science 198 (1977) 293-295.

[43] K.Q. Zhang, X.Y. Liu, Determination of elastic constants of two-dimensional closepacked colloidal crystals, Langmuir 25 (2009) 5432-5436.

[44] P. Wette, H.J. Schöpe, J. Liu, T. Palberg, Characterisation of colloidal solids, Prog Colloid Polym. Sci. 123 (2004) 264-268.

[45] J.L. Harland, W. vanMegen, Crystallization kinetics of suspensions of hard colloidal spheres, Phys. Rev. E 55 (1997) 3054-3067.

[46] H.W. Zhou, S.H. Xu, Z.W. Sun, X. Du, L.X. Liu, Kinetics study of crystallization with the disorder-bcc-fcc phase transition of charged colloidal dispersions, Langmuir 27 (2011) 7439-7445.

[47] S. Iacopini, T. Palberg, H.J. Schope, Ripening-dominated crystallization in polydisperse hard-sphere-like colloids, Phys. Rev. E 79 (2009).

[48] I.M. Lifshitz, V.V. Slyozov, The kinetics of precipitation from supersaturated solid solutions, J. Phys. Chem. Solids 19 (1961) 35-50. 